\title{
Dose specification in External Beam Radiotherapy for CyberKnife and VMAT techniques applied to a case of prostate cancer
}

\author{
Krzysztof Ślosarek ${ }^{1,2}$, Joanna Kopczyńska ${ }^{1}$, Wojciech Osewski ${ }^{3}$
}

Recent technological development in radiotherapy allows to introduce new irradiation techniques implemented on the conventional accelerators and on the machines such as CyberKnife (CK). These significantly changes the philosophy of planning and execution of radiotherapy. One of the fundamental concepts in radiotherapy is to define the therapeutic dose. It can be defined in the point, at the selected isodose, as an average value, or combined with the volume. We present the case of prostate cancer patient irradiated using CK machine and classic accelerator (VMAT). The differences in dose distribution and its value are shown. The analysis indicates that the average dose in Planning Treatment Volume (PTV) is a useful parameter during comparison of the dose distributions realized on machines of different type.

NOWOTWORY J Oncol 2016; 66, 5: 375-380

Key words: prescribed dose, normalization, dose volume histogram (DVH)

\section{Aim}

To choose those prescribed dose values that enable the same bioequivalent dose to be administered when using two different radiation techniques.

\section{Background}

Extra cranial stereotactic radiotherapy is now rendered possible by rapid technological developments when twodimensional (2D) treatment planning became converted to three-dimensions (3D), followed by the introduction of Intensity Modulated Radiotherapy (IMRT), Volumetric Modulated Arc Therapy (VMAT) and CyberKnife (Accuray, Sunnyvale, CA, USA) [1-3]. The therapeutic dose is one of the basic concepts in radiotherapy. Modern irradiation techniques have now enabled the dose to be escalated to the PTV (Planned Treatment Volume) whilst simultaneously protecting/sparing OaRs (Organs at Risk) [4]. Nevertheless, knowing the fractional or total dose for a given field is insufficient, because they can be defined in different ways: as a point, by a selected isodose, ' $X$ ' percent of the prescribed dose coverage [5], ' $Y$ ' percent of the treated volume or the mean, modal or median dose to the PTV.

Dose distributions generated by modern therapeutic instrumentation are intentionally inhomogeneous and are now different to those previously accepted [6-9]. For example, the concept of isocenter, by which means the dose is commonly defined, is absent for the CyberKnife (CK) instrument. For classical accelerators, using IMRT and VMAT dynamic techniques, the shape of the beam changes during exposition. It is therefore reasonable to define doses in volumes, but not at a point because this can become obscured by the moving collimator leaves. Usually, the irradiation is performed on only one type of therapeutic instrument. In some cases however, treatment is required to be continued on through using a different instrument, where information concerning the absorbed dose and the delivery location becomes important [5]. Dose distributions obtained using CK instruments definitely differ from those

\footnotetext{
${ }^{1}$ Radiotherapy Planning Department, Maria Sklodowska-Curie Memorial Cancer Center and Institute of Oncology Gliwice Branch, Poland

${ }^{2}$ Humanitas University, Sosnowiec, Poland

${ }^{3}$ Department of Radiotherapy, Maria Sklodowska-Curie Memorial Cancer Center and Institute of Oncology Gliwice Branch, Poland
} 

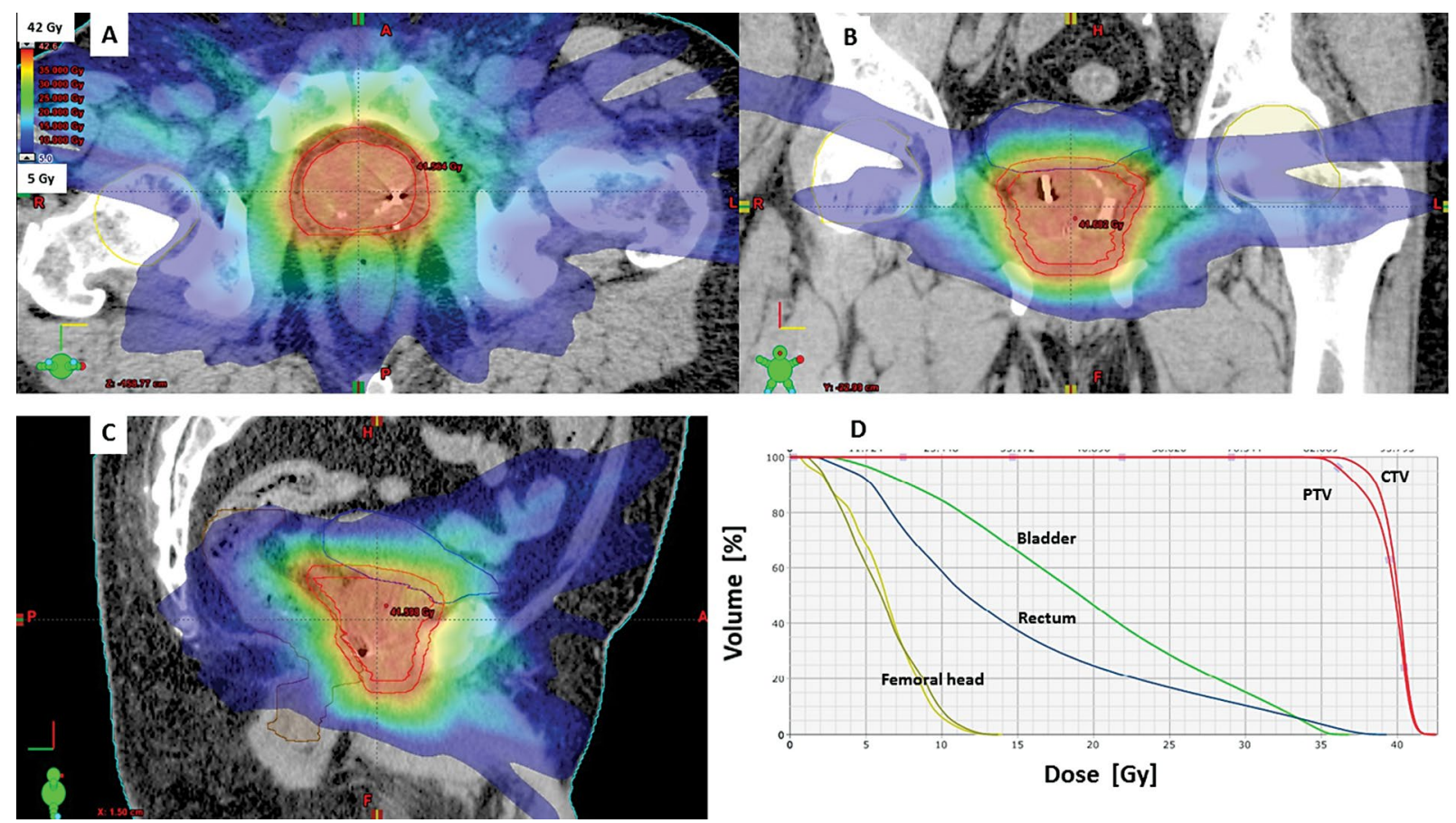

Figure 1. Planned dose distribution for the CyberKnife. Plane $\mathbf{A}-$ transversal, $\mathbf{B}-$ frontal and $\mathbf{C}-$ sagittal together with $\mathbf{D}-\mathrm{DVH}$. The total $36.25 \mathrm{~Gy}$ dose was defined at the $85 \%$ isodose. Blue indicates a dose of $5 \mathrm{~Gy}$ and red of $41 \mathrm{~Gy}$

used for the IMRT and VMAT dynamic techniques. For the CK, doses are defined to isodoses (ranging 70-90\%) whereas for conventional accelerators in dynamic techniques they are defined by the volume ( $95 \%$ of the prescribed dose at $100 \%$ of treated volume) [10-12].

\section{Material and methods}

This article presents the case of an irradiated patient with prostate cancer that underwent CK treatment. A total treatment dose of $36.25 \mathrm{~Gy}$ was given as five fractions (7.25 Gy) defined to the $85 \%$ isodose [13] and had been planned using the MultiPlan v. 4.1 (Accuray, Sunnyvale, CA, USA). The overall treatment time was 10 days. Organs at Risk: bladder, rectum and femoral heads, those volumes were reduced by the common part with PTV.

According to treatment protocols, 13 planned doses to the bladder cannot exceed: $\mathrm{V}_{18 \mathrm{~Gy}}-55 \%, \mathrm{~V}_{29 \mathrm{~Gy}}-25 \%$, $\mathrm{V}_{32,6 \mathrm{~Gy}}-15 \%$ and $\mathrm{V}_{23,25 \mathrm{~Gy}}-10 \%$, for the bladder these being: $\mathrm{V}_{18 \mathrm{~Gy}}-55 \%, \mathrm{~V}_{29 \mathrm{~Gy}}-20 \%, \mathrm{~V}_{32,6 \mathrm{~Gy}}-10 \%$ and $\mathrm{V}_{23,25 \mathrm{~Gy}}$ $-5 \%$ for the rectum: $\mathrm{V}_{25 \mathrm{~Gy}}-45 \%$ and for the femoral heads the maximum dose cannot exceed $120 \%$ of the prescribed dose.

Stereotactic radiotherapy for prostate cancer can also be delivered by the VMAT technique using classical accelerators [14], where the dose distribution is calculated by the Eclipse TPS v. 10 (Varian Medical Systems, Palo Alto, CA, USA). A dose fraction of 7.25 Gy was defined as $95 \%$ of the prescribed dose to $100 \%$ of the treated volume. Dose limits to the OaRs were kept to the same level as for the CyberKnife technique.

Comparisons of dose distribution were performed on the Eclipse TPS. In-house 'DDcon' software was used for exporting the CK dose distribution from the Multiplan TPS to the Eclipse TPS [15]. In order to assess the differences between doses to the CTV (Clinical Target Volume) and PTV, in the form of dose sets for both techniques, the exported differential DVH files were compared by the t-test for independent samples using STATISTICA v. 10 software; statistical significance being taken as $\leq 0.05$. In-house RPIWin software was also used to calculate the RPI coefficient [16] for determining the differences in dose distribution between both techniques; this being achieved by using cumulative DVH's for both techniques. Finally, bioequivalent doses (Diso2Gy) were calculated for both treatment methods, and based on the mean dose they were normalised to the 2Gy fraction using a linear-quadratic model (LQ). Bioequivalent doses directly affect the TCP.

\section{Results}

Figure 1 shows the calculated CK dose distribution according to the previously described scheme along with the Dose Volume Histograms (DVH) for the CTV, PTV, rectum, bladder and femoral heads structures. This treatment plan was accepted and performed on the CK.

Figure 2 shows the calculated dose distribution for the same patient, but using the VMAT technique with classical 

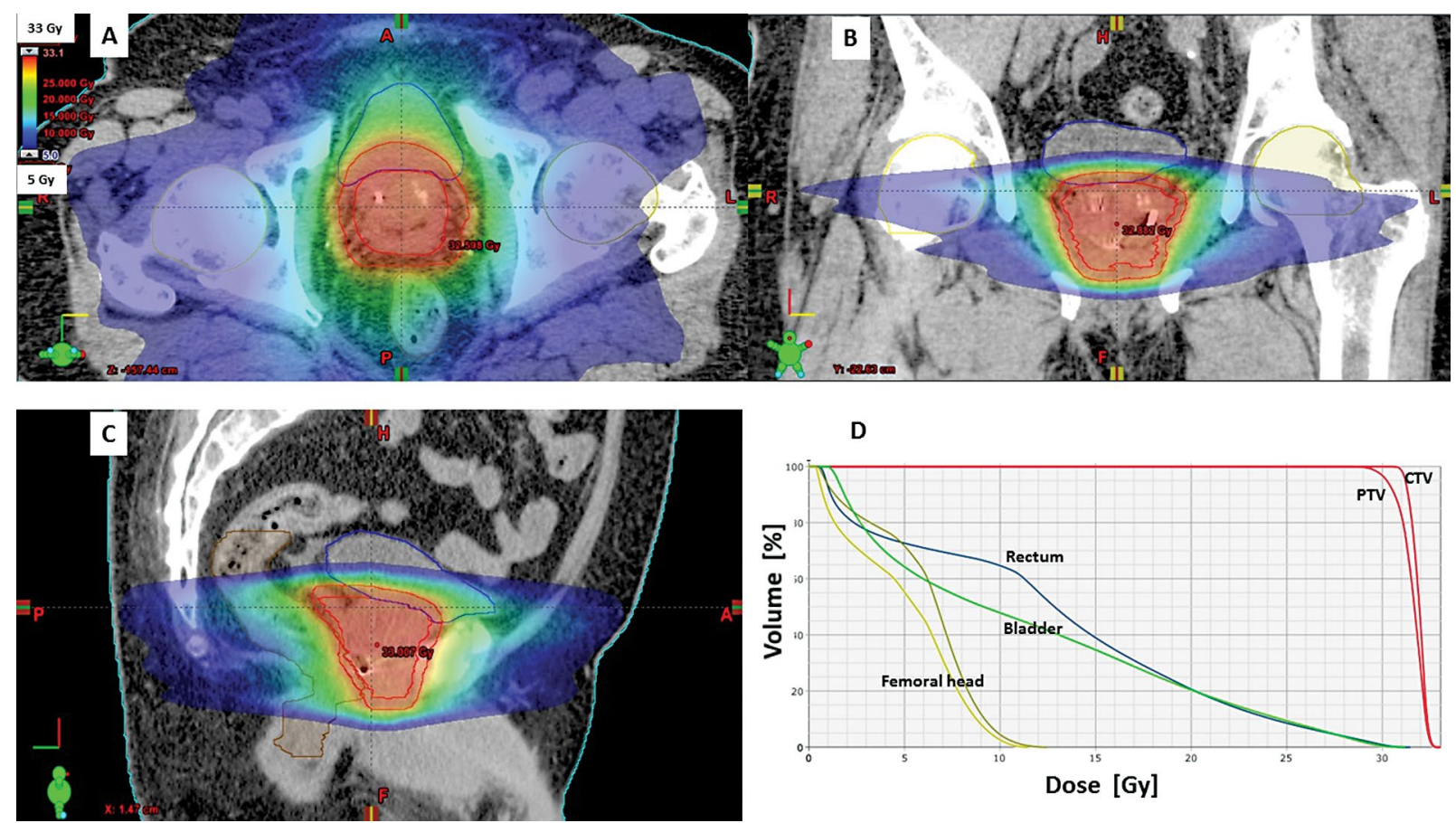

Figure 2. Planned dose distribution for the VMAT technique. Plane $\mathbf{A}$ - transversal, $\mathbf{B}-$ frontal and $\mathbf{C}-$ sagittal together with $\mathbf{D}-\mathrm{DVH}$. The total dose 36.25 Gy was defined at the $95 \%$ isodose which covers $100 \%$ of the CTV. Blue indicates a dose of 5 Gy and red of $41 \mathrm{~Gy}$

accelerators (Linac CL23EX Varian Medical Systems, Palo Alto, CA, USA). The treatment plan consisted of 3 arcs and $20 \mathrm{MV}$ beams. The dose was defined at $95 \%$ of the planned dose in $100 \%$ of the irradiated volume.

After analysing dose distributions for both DVH-based treatment plans, the mean, median, modal, minimum and maximum dose values demonstrated considerable variation; as shown in Table I.

The planned total dose (36.5 Gy) leads to delivering a maximum dose of $43 \mathrm{~Gy}$ to the CTV for the CK and $39 \mathrm{~Gy}$ for the VMAT techniques. These differences are especially pronounced in the differential histogram. The mean doses to the CTV and for the CK and VMAT techniques were respectively $39.9 \mathrm{~Gy}$ and $36.2 \mathrm{~Gy}$, whilst those for the PTV were respectively $40.6 \mathrm{~Gy}$ and $35.8 \mathrm{~Gy}$. The data are shown in Figure 3.

Upon analysing simultaneous doses to critical organs, differences between the CK and VMAT method are small; as shown in Figure 4.

Significant statistical differences were found between doses to the CTV ( $p=0.0002$, t-test for independent samples) and to the PTV ( $p=0.0262)$, when comparing the dose distributions for CK (dose defined at the $85 \%$ isodose) with those for the VMAT ( $95 \%$ of planned dose to $100 \%$ of the CTV volume). The minimum, maximum and mean values are presented in Table $\mathrm{l}$. Correspondingly, there were no significant differences for the bladder ( $p=0.9344)$, rectum ( $p=0.2027)$ nor in the femoral heads; right $(p=0.3036)$ and left $(p=0.5252)$.

Table I. Total doses; minimum, maximum and mean for CKand VMAT treatment techniques. Therapeutic doses were defined according to recommendations for both methods as: $85 \%$ of the dose to the CTV for CK and $95 \%$ of the dose to $100 \%$ of the CTV for VMAT. Bold indicates values that require to be taken into account when evaluating the treatment plan

\begin{tabular}{|c|c|c|c|c|c|c|}
\hline & \multicolumn{6}{|c|}{ Total dose [Gy] } \\
\hline & \multicolumn{2}{|c|}{ Minimum } & \multicolumn{2}{|c|}{ Maximum } & \multicolumn{2}{|c|}{ Mean } \\
\hline & CK & VMAT & CK & VMAT & CK & VMAT \\
\hline CTV & 35.2 & 34.4 & 42.5 & 37.5 & 39.9 & 36.2 \\
\hline PTV & 34.2 & 31.1 & 44 & 37.5 & 40.6 & 35.8 \\
\hline Rectum & 1.5 & 0.6 & 39.3 & 35.7 & 14.6 & 14.3 \\
\hline Bladder & 2.5 & 1.1 & 36.9 & 35.4 & 19.5 & 12.9 \\
\hline Femoral head - left & 0.6 & 0.4 & 14 & 13.1 & 6.2 & 5.7 \\
\hline Femoral head - right & 1 & 0.5 & 13.7 & 14.2 & 6.1 & 6.9 \\
\hline
\end{tabular}




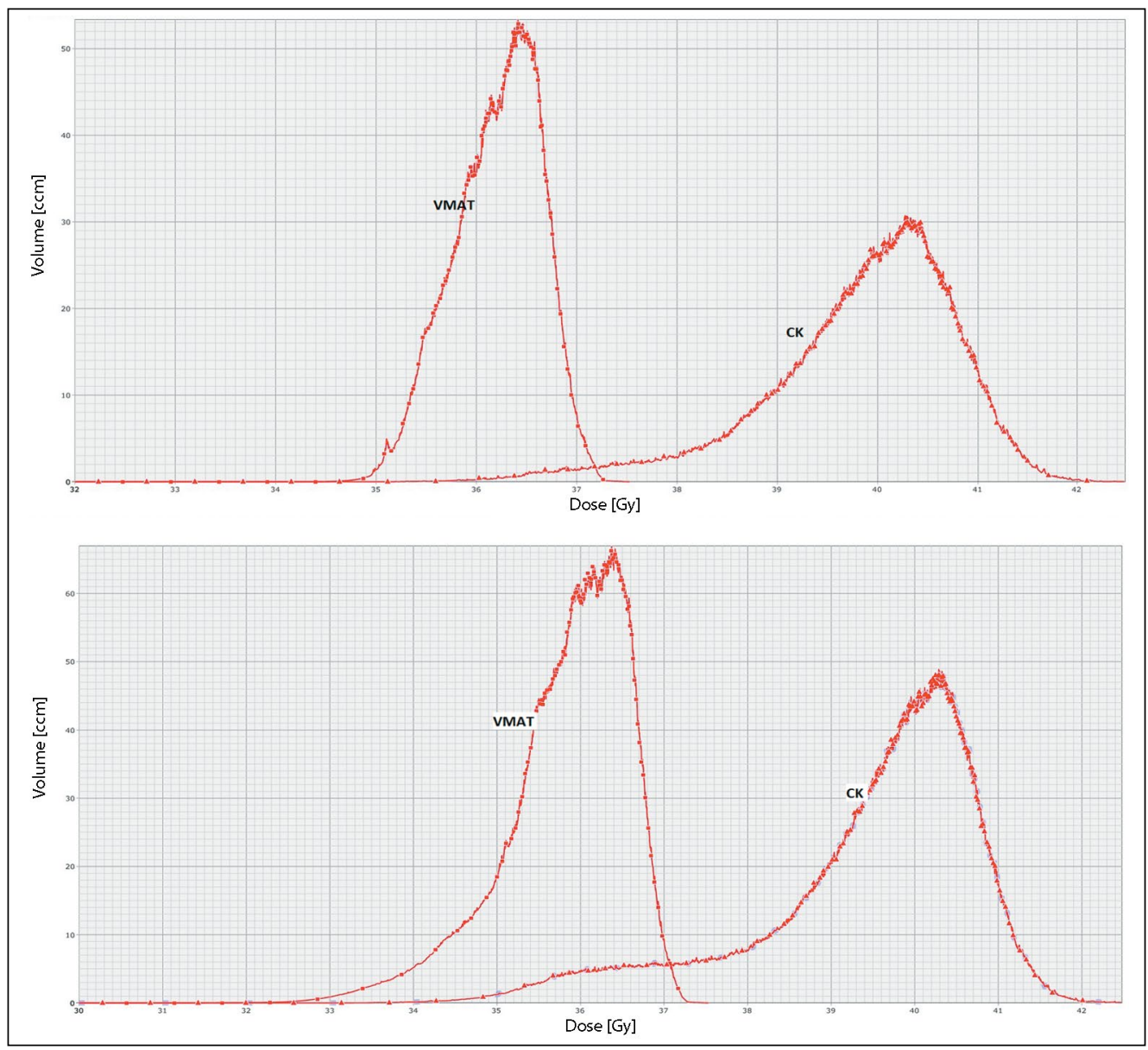

Figure 3. Differential DVHs for the CK and VMAT irradiation techniques for a planned dose of $36.25 \mathrm{~Gy}: \mathbf{A}$ - for the CTV and B - for the PTV; the mean dose for the CK technique being greater than for VMAT as on the graph

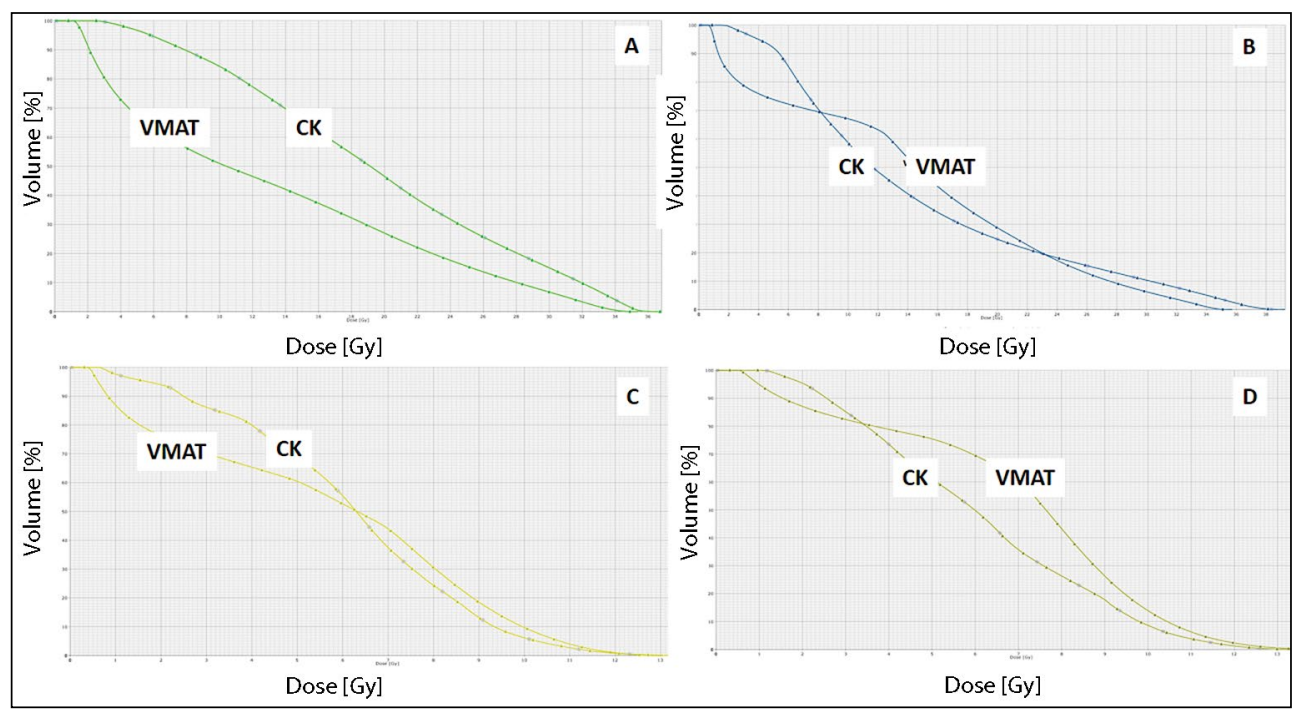

Figure 4. Dose distribution obtained by the CK and VMAT techniques for Organs at Risk: A — bladder, B - rectum, C - left femoral head and D - right femoral head 


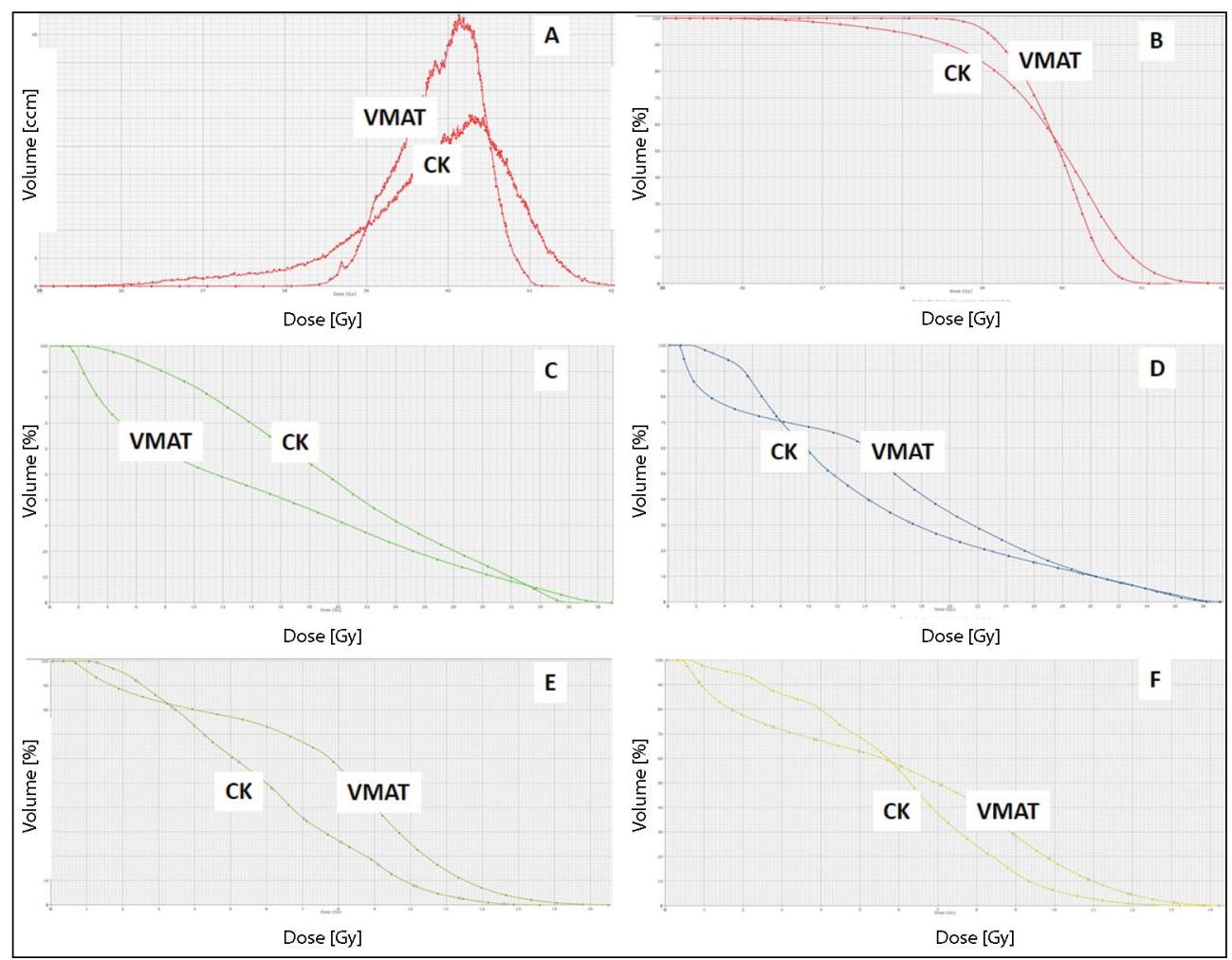

Figure 5. Differential DVH's for CK and VMAT technique; A - CTV, B - PTV, C — bladder, D - rectum, E - right femoral head, F — left femoral head

The calculated RPI coefficient for the CK was 0.5095 and 0.5443 for the VMAT meaning that the treatment plan using the latter technique better met our expectations. Nevertheless, it can be said that the dose distributions calculated for both methods are comparable from the viewpoint of treatment planning.

According to the LQ model, the calculated bioequivalent dose (to a 2 Gy fractional dose) for CK and VMAT are however different, being respectively 99.6 Gy2 vs 83.6 Gy2 for the CTV and 102.7 Gy2 vs 82.0 Gy2 for the PTV.

It should also be emphasised, that the doses recorded in the treatment cards were $36.25 \mathrm{~Gy}$ for both CK and the classical accelerator.
In order to make dose distributions comparable between irradiation techniques for the CTV and PTV, along with the delivered bioequivalent doses, a mean dose value of 39.9 Gy to the CTV was chosen for the CK technique. A re-normalisation thus became necessary for the VMAT technique, such that its value was equal to the mean CK dose, as shown in Figure 5.

Table II compares the minimum, maximum and mean doses for both techniques where the VMAT doses were renormalised to CK mean dose. Red denotes values requiring to be taken into account when evaluating the treatment plan.

Re-normalised dose values were likewise calculated to allow the comparison of dose distributions between methods

Table II. Total doses, minimum, maximum and mean values for the CK and VMAT techniques, where the mean doses for VMAT were normalised to the mean CK value. Bold denotes values that require special attention when evaluating the treatment plan

\begin{tabular}{|c|c|c|c|c|c|c|c|}
\hline & \multicolumn{7}{|c|}{ Total dose DGy2 $\left(\mathrm{Gy}_{2}\right)$} \\
\hline & \multicolumn{3}{|c|}{ Minimum } & \multicolumn{2}{|c|}{ Maximum } & \multicolumn{2}{|c|}{ Mean } \\
\hline & $\alpha / \beta[\mathrm{Gy}]$ & CK & VMAT & CK & VMAT & CK & VMAT \\
\hline CTV & 1.5 & 85.9 & 82.4 & 121.4 & 96.4 & 108.1 & 90.4 \\
\hline PTV & 2 & 75.6 & 63.9 & 118.8 & 89.1 & 102.7 & 82.0 \\
\hline Rectum & 15 & 1.4 & 0.5 & 52.8 & 46.5 & 15.4 & 15.0 \\
\hline Bladder & 15 & 2.3 & 1.0 & 48.6 & 46.0 & 21.7 & 13.3 \\
\hline Femoral head - left & 15 & 0.5 & 0.4 & 14.7 & 13.6 & 5.9 & 5.4 \\
\hline Femoral head - right & 15 & 0.9 & 0.4 & 14.3 & 14.9 & 5.8 & 6.6 \\
\hline
\end{tabular}


$(100 \%=$ mean dose). No significant differences were found for the CTV ( $p=0.53330)$, PTV ( $p=0.612088)$ nor for the OaRs (Bladder, Rectum , Femoral Heads; $p=0.075$ ). The calculated RPI factor for the VMAT was 0.5673 , thereby indicating that the relationship between the dose to the CTV/PTV and OaRs is improved in terms of the assumptions made to dose distribution. The calculated Bioequivalent Doses, (based on the LQ model), to the CTV/PTV were 99.6/102.7 Gy2 and 99.6/97.8 Gy2 for CK and VMAT, respectively.

\section{Conclusions}

Upon comparing dose distributions between two different irradiation methods, the bioequivalent doses may be different irrespective of whether the therapeutic dose and the fractionation method have been declared the same. The mean dose value should therefore be taken into account for the therapeutic dose. This is of course does not accord with the reported recommendations, nevertheless such information could prove valuable when comparing different irradiation techniques.

For the presented case, the fractional dose for the VMAT technique should be increased from 7.25 to 7.99 Gy thus making the bioequivalent doses comparable for the two studied methods. The described situation provides an example which not only applies to different therapeutic instruments, but also to different irradiation techniques used on the same accelerator e.g. CRT vs IMRT/VMAT.

\section{Conflicts of interest: none declared}

\section{Joanna Kopczyńska, MD, PhD}

Department of Radiotherapy

Maria Sklodowska-Curie Memorial Cancer Center

and Institute of Oncology

Gliwice Branch

Wybrzeże AK 15, 44-100 Gliwice, Poland

e-mail:joanna.bystrzycka@io.gliwice.pl

Received: 19 Apr 2016

Accepted: 3 Jul 2016

\section{References}

1. Yu CX, Tang G. Intensity modulated arc therapy: principles, technologies and clinical implementation. Phys Med Biol 2011; 56: R31-R54.

2. Yuatsev S, Muren L, Thwaites D. Treatment planning studies in radiotherapy. Radioth Oncol 2013; 109, 342-243.

3. King C, Lehmann J, Adler J et al. CyberKnife radiotherapy for localized prostate cancer: rationale and technical feasibility, technology in cancer research \& treatment. Technical Cancer Res Treat 2003; 2: 25-30.

4. Murray L, Cosgrove V, Lilley J et al. Developing a class solution for Prostate Stereotactic Ablative Body Radiotherapy (SABR) using Volumetric Modulated Arc Therapy (VMAT). Radioth Oncol 2014, 110, 298-302.

5. Williams G, Tobler M, Leavitt D. Pitfalls in normalization for intensitymodulated radiation therapy planning. Med Dosim 2005; 30: 194-200.

6. Ślosarek K, Tarnawski R, Maciejewski B. Is there need for inhomogeneous dose distribution? New concept of IMRS. 7th International Meeting on Progress in Radio-Oncology (ICRO/ÖGRO 7), Salzburg 2002.

7. Maciejewski B, Skołyszewski J, Majewski $S$ et al. Applicability of the biological isodoses „IzoBioGy-2" for tumour and normal tissue response for fractionated radiotherapy. Nowotwory 1988; 37: 241-249.

8. Fowler J, Chappell R, Ritter M. Is alpha/beta for prostate tumours really low? Inter J Radiat Oncol Biol Phys 2001; 50: 1021-1031.

9. Ślosarek K, Rembielak A, Grządziel A et al. New possibility In IMRT treatment planning, $9^{\text {th }}$ Varian European Users Meeting, Sardinia, 7-9 June, 2001.

10. Ślosarek K, Zajusz A, Szlag M. Comparison of traditional and simultaneous IMRT boost technique basing on therapeutic gain calculation. Med Dosim 2008; 33: 299-302.

11. Wu Q, Mohan R, Niemirko A et al. Optimization of intensity modulated radiotherapy plans based on the equivalent uniform dose. Inter J Radiat Oncol Biol Phys 2002; 52: 224-235.

12. A Prescription Template for RTOG Protocols, (http://www.rtog.org/ LinkClick.aspx?fileticket=v-QYwJs2HaU\%3d\&tabid=197).

13. Ferro $M$, Cilla $S, M a c c h i a ~ G$ et al. On the cutting edge of intensity modulated radiotherapy and simultaneous integrated boost (IMRT-SIB): The case of a patient with 8 brain metastases. Rep Practi Oncol Radiother 2015; 20: 316-319.

14. IRCU 83

15. Głowacki G, Miszczyk L, Blamek S et al. Protocol of radical fractionated stereotactic radiosurgery CyberKnife, patients with prostate cancer. Center of Oncology - MSC Memorial Institute, Branch Gliwice, 2013.

16. Ślosarek K, Osewski W, Grzadziel A et al. Integral dose: Comparison between four techniques for prostate radiotherapy. Rep Pract Oncol Radioth 2014; 20: 99-103.

17. Osewski W, Ślosarek K, Karaszewska B. Dose distribution transfer from CyberKnife to Varian treatment planning system. J Phys: Conf Series 2014; 489: 012056

18. Ślosarek K, Grządziel A. Szlag M et al. Radiation Planning Index for dose distribution evaluation in stereotactic radiotherapy, Rep Pract Oncol Radiother 2008; 13: 182-186.

19. Maciejewski B, Ślosarek K. Zastosowanie w radioterapii wzorów dawek równoważnych - NSD, TDF, CRE. Centrum Onkologii — Instytut im. M. Skłodowskiej-Curie, Gliwice, 1979. 